\title{
Synthesis of a Phosphorus-Containing Hybrid Porphyrin
}

\author{
Yoshihiro Matano,* Takashi Nakabuchi, Tooru Miyajima, Hiroshi Imahori, and Haruyuki Nakano \\ Department of Molecular Engineering, Graduate School of Engineering, Kyoto University, Nishikyo- \\ ku, Kyoto 615-8510, Japan and Department of Chemistry, Graduate School of Sciences, Kyushu \\ University, Fukuoka 812-8581, Japan \\ matano@scl.kyoto-u.ac.jp \\ FAX: $+81-75-383-2571$
}

\section{Contents:}

General:

SI-2

Synthesis and Characterization of 2-6:

SI-2

Figures S1 S4 ( ${ }^{1} \mathrm{H}$ NMR Charts):

SI-5

Density Functional Theory Calculations on Model Complexes and Figures S5, S6: SI-9 


\section{General}

All melting points are uncorrected. ${ }^{1} \mathrm{H},{ }^{13} \mathrm{C}\left\{{ }^{1} \mathrm{H}\right\}$, and ${ }^{31} \mathrm{P}\left\{{ }^{1} \mathrm{H}\right\}$ NMR spectra were recorded using $\mathrm{CDCl}_{3}$ as the solvent unless otherwise noted. The ${ }^{1} \mathrm{H}$ NMR spectra of $\mathbf{2 , 4 , 5}$, and $\mathbf{6}$ are shown in Figures S1-S4. Chemical shifts are reported as the relative value vs. tetramethylsilane $\left({ }^{1} \mathrm{H}\right)$ and phosphonic acid $\left({ }^{31} \mathrm{P}\right)$. Mass spectra were measured using CHCA (MALDI-TOF), $m$-nitrobenzyl alcohol (FAB), or $m$-nitrophenyl octyl ether (HR-FAB) as a matrix. All solvents were distilled from sodium benzophenone ketyl (ether, THF) or calcium hydride $\left(\mathrm{CH}_{2} \mathrm{Cl}_{2}\right.$, toluene) before use. All the reactions were performed under an argon or nitrogen atmosphere. Column chromatography was performed on silica gel or activated alumina. Compounds $\mathbf{1}^{1}$ and $\mathbf{3}^{2}$ were prepared according to the reported procedures.

\section{Synthesis and Characterization of 2-6}

Phosphatripyrrane 2: To a mixture of 1 (1.9 g, $6.5 \mathrm{mmol})$ and pyrrole (35 $\mathrm{mL}, 510 \mathrm{mmol})$ was added $\mathrm{BF}_{3} \cdot \mathrm{OEt}_{2}(4.2 \mathrm{~mL}, 33 \mathrm{mmol})$ at $80{ }^{\circ} \mathrm{C}$. After stirring for $1.5 \mathrm{~h}$ at the same temperature, the second portion of $\mathrm{BF}_{3} \bullet \mathrm{OEt}_{2}(4.9 \mathrm{~mL}, 39 \mathrm{mmol})$ was added, and the mixture was stirred for an additional $1 \mathrm{~h}$. After cooling to room temperature, a saturated $\mathrm{NaHCO}_{3}$ solution $(20 \mathrm{~mL})$ and $\mathrm{CH}_{2} \mathrm{Cl}_{2}(50 \mathrm{~mL})$ were added. The organic phase was separated, dried over $\mathrm{Na}_{2} \mathrm{SO}_{4}$, and evaporated. The products were subjected to silica gel column chromatography $\left(\mathrm{CH}_{2} \mathrm{Cl}_{2}\right)$ repeatedly and the fraction of $R_{\mathrm{f}}=0.3$ was collected and washed with $\mathrm{MeOH}$ to give 2 as a colorless solid (350 mg, 14\%): $\mathrm{Mp} 141-143{ }^{\circ} \mathrm{C} ;{ }^{1} \mathrm{H}$ NMR $\delta 1.98-2.05(\mathrm{~m}, 2 \mathrm{H}), 2.25-2.40(\mathrm{~m}, 4 \mathrm{H}), 3.48-3.53(\mathrm{~m}, 4 \mathrm{H}), 5.85-5.87(\mathrm{~m}, 2 \mathrm{H})$, 5.96-5.98 (m, 2H), 6.57-6.58 (m, 2H), 7.39-7.43 (m, 2H), 7.48-7.50 (m, 1H), 7.71-7.75 (m, 2H), 8.77 (br, 2H); ${ }^{13} \mathrm{C}\left\{{ }^{1} \mathrm{H}\right\} \operatorname{NMR} \delta 23.7\left(\mathrm{~d},{ }^{2} J_{\mathrm{P}-\mathrm{C}}=15.6 \mathrm{~Hz}\right), 26.7\left(\mathrm{~d},{ }^{4} J_{\mathrm{P}-\mathrm{C}}=1.9 \mathrm{~Hz}\right), 26.8\left(\mathrm{~d},{ }^{3} J_{\mathrm{P}-\mathrm{C}}=11.8 \mathrm{~Hz}\right), 106.3(\mathrm{~s})$, $107.7(\mathrm{~s}), 117.3(\mathrm{~s}), 127.1\left(\mathrm{~d},{ }^{1} J_{\mathrm{P}-\mathrm{C}}=69.0 \mathrm{~Hz}\right), 127.2\left(\mathrm{~d},{ }^{3} J_{\mathrm{P}-\mathrm{C}}=3.7 \mathrm{~Hz}\right), 128.8\left(\mathrm{~d},{ }^{2} J_{\mathrm{P}-\mathrm{C}}=12.5 \mathrm{~Hz}\right), 130.3$

\footnotetext{
${ }^{1}$ Matano, Y.; Miyajima, T.; Nakabuchi, T.; Matsutani, Y.; Imahori, H. J. Org. Chem. 2006, 71, 5792. 2 (a) Chadwick, D. J.; Willbe, C. J. Chem. Soc., Pekin Trans. 1 1977, 887. (b) Jang, Y.-S.; Kim H.-J.; Lee, P.-H.; Lee, C.-H. Tetrahedron Lett. 2000, 41, 2919.
} 
$\left(\mathrm{d},{ }^{3} J_{\mathrm{P}-\mathrm{C}}=11.9 \mathrm{~Hz}\right), 132.0\left(\mathrm{~d},{ }^{4} J_{\mathrm{P}-\mathrm{C}}=2.5 \mathrm{~Hz}\right), 157.8\left(\mathrm{~d},{ }^{2} J_{\mathrm{P}-\mathrm{C}}=25.5 \mathrm{~Hz}\right)$; The ipso carbon of the P-phenyl group could not be assigned; ${ }^{31} \mathrm{P}\left\{{ }^{1} \mathrm{H}\right\}$ NMR $\delta$ 72.2; MS (FAB) $m / z 391\left(\mathrm{M}^{+}\right)$. HR-FAB-MS: Calcd for $\mathrm{C}_{23} \mathrm{H}_{24} \mathrm{~N}_{2} \mathrm{PS}\left(\mathrm{M}^{+}\right), 391.1398 ;$ Found, 391.1398 .

$\sigma^{4}$-Porphyrinogen 4: $\mathrm{A} \mathrm{CH}_{2} \mathrm{Cl}_{2}$ solution $(530 \mathrm{~mL})$ containing $2(370 \mathrm{mg}, 0.95 \mathrm{mmol})$ and 3 (280 mg, $0.95 \mathrm{mmol})$ was bubbled with $\mathrm{N}_{2}$ for $30 \mathrm{~min} . \mathrm{BF}_{3} \bullet \mathrm{OEt}_{2}(0.12 \mathrm{~mL}, 0.95 \mathrm{mmol})$ was added to the solution, and the mixture was then stirred for $30 \mathrm{~min}$ at room temperature. The resulting mixture was washed with distilled water $(2 \times 500 \mathrm{~mL})$, dried over $\mathrm{Na}_{2} \mathrm{SO}_{4}$, and evaporated. The crude products were subjected to silica gel column chromatography (hexane $/ \mathrm{CH}_{2} \mathrm{Cl}_{2}=1 / 1$ ) to give $\sigma^{4}$-porphyrinogen 4 as a mixture of three diastereomers $\left(R_{\mathrm{f}}=0.5-0.6 ; 220 \mathrm{mg}, 36 \%\right)$. The ${ }^{1} \mathrm{H}$ and ${ }^{31} \mathrm{P}$ NMR spectra indicated that three major diastereomers $(\mathbf{A}, \mathbf{B}, \mathbf{C})$ were included in a ratio of 6:5:3. ${ }^{1} \mathrm{H}$ NMR Diastereomer A: $\delta 1.76-1.90$ (m, 1H), 2.00-2.19 (m, 1H), 2.20-2.46 (m, 4H), 3.42-3.49 (m, 4H), 5.37-5.39 (m, 1H), $5.46(\mathrm{~s}, 1 \mathrm{H}), 5.54\left(\mathrm{~d},{ }^{4} \mathrm{~J}=1.6 \mathrm{~Hz}, 1 \mathrm{H}\right), 5.75-5.78(\mathrm{~m}, 1 \mathrm{H}), 5.82-5.83(\mathrm{~m}, 1 \mathrm{H})$, 5.87-5.89 (m, 1H), $6.19\left(\mathrm{dd}, J=3.6 \mathrm{~Hz},{ }^{4} J=1.6 \mathrm{~Hz}, 1 \mathrm{H}\right), 6.82(\mathrm{~d}, J=3.6 \mathrm{~Hz}, 1 \mathrm{H}), 7.18-7.39$ (m, 10H), 7.47-7.49 (m, 2H), 7.52-7.56 (m, 1H), 7.82-7.87 (m, 2H), 9.38 (br, 1H), 9.74 (br, 1H);

Diastereomer B: $\delta$ 1.76-1.90 (m, 1H), 2.00-2.19 (m, 1H), 2.20-2.46 (m, 4H), 3.42-3.49 (m, 4H), 5.54 $(\mathrm{s}, 2 \mathrm{H}), 5.77-5.78(\mathrm{~m}, 2 \mathrm{H}), 5.87-5.88(\mathrm{~m}, 2 \mathrm{H}), 6.14(\mathrm{~s}, 2 \mathrm{H}), 7.18-7.39(\mathrm{~m}, 10 \mathrm{H}), 7.47-7.49(\mathrm{~m}, 2 \mathrm{H})$, 7.52-7.56 (m, 1H), 7.82-7.87 (m, 2H), $9.61(\mathrm{br}, 2 \mathrm{H})$; Diastereomer C: $\delta 1.76-1.90(\mathrm{~m}, 1 \mathrm{H}), 2.00-2.19$ (m, 1H), 2.20-2.46 (m, 4H), 3.42-3.49 (m, 4H), 5.29-5.32 (m, 2H), 5.59 (s, 2H), 5.79-5.81 (m, 2H), $6.90(\mathrm{~s}, 2 \mathrm{H}), 7.18-7.39(\mathrm{~m}, 10 \mathrm{H}), 7.47-7.49(\mathrm{~m}, 2 \mathrm{H}), 7.52-7.56(\mathrm{~m}, 1 \mathrm{H}), 7.82-7.87(\mathrm{~m}, 2 \mathrm{H}), 9.51$ (br, $2 \mathrm{H}) ;{ }^{31} \mathrm{P}\left\{{ }^{1} \mathrm{H}\right\}$ NMR $\delta$ 72.6, 72.7, 72.8; MS (MALDI-TOF) $m / z 650\left(\mathrm{M}^{+}\right)$.

$\sigma^{3}$-Porphyrinogen 5: To a toluene solution $(40 \mathrm{~mL})$ of $\mathbf{4}(370 \mathrm{mg}, 0.57 \mathrm{mmol})$ was added $\mathrm{P}\left(\mathrm{NMe}_{2}\right)_{3}$ $(0.66 \mathrm{~mL}, 3.6 \mathrm{mmol})$, and the mixture was then stirred under reflux for $23 \mathrm{~h}$. The resulting mixture was concentrated under reduced pressure and subjected to silica gel column chromatography (hexane $\left./ \mathrm{CH}_{2} \mathrm{Cl}_{2}=2 / 1\right)$ to give 5 as a mixture of diastereomers $\left(R_{\mathrm{f}}=0.3-0.4 ; 330 \mathrm{mg}\right.$, 93\%): The ratio 
of the three diastereomers $(\mathbf{A}, \mathbf{B}, \mathbf{C})$ was found to be 8:5:4. ${ }^{1} \mathrm{H}$ NMR Diastereomer A: $\delta$ 2.10-2.35 (m, 4H), 2.50-2.63 (m, 2H), 3.40-3.45 (m, 2H), 3.68-3.78 (m, 2H), $5.55(\mathrm{~s}, 1 \mathrm{H}), 5.60(\mathrm{~s}, 1 \mathrm{H}), 5.72-5.76$ $(\mathrm{m}, 2 \mathrm{H}), 5.77-5.81(\mathrm{~m}, 1 \mathrm{H}), 5.82-5.86(\mathrm{~m}, 1 \mathrm{H}), 6.55(\mathrm{~d}, J=3.6 \mathrm{~Hz}, 1 \mathrm{H}), 6.67(\mathrm{~d}, J=3.6 \mathrm{~Hz}, 1 \mathrm{H})$, 7.22-7.30 (m, 15H), 8.20 (br, 1H), 8.35 (br, 1H); Diastereomer B: $\delta 2.10-2.35(\mathrm{~m}, 4 \mathrm{H}), 2.50-2.63(\mathrm{~m}$, 2H), 3.40-3.45 (m, 2H), 3.68-3.78 (m, 2H), $5.60(\mathrm{~s}, 2 \mathrm{H}), 5.65-5.69(\mathrm{~m}, 2 \mathrm{H}), 5.78-5.82(\mathrm{~m}, 2 \mathrm{H}), 6.67$ (s, 2H), 7.22-7.30 (m, 15H), 8.27 (br, 2H); Diastereomer C: $\delta$ 2.10-2.35 (m, 4H), 2.50-2.63 (m, 2H), 3.40-3.45 (m, 2H), 3.68-3.78 (m, 2H), 5.60-5.62 (m, 4H), 5.78-5.81 (m, 2H), $6.76(\mathrm{~s}, 2 \mathrm{H}), 7.22-7.30$ (m, 15H), 8.21 (br, $1 \mathrm{H}) ;{ }^{31} \mathrm{P}\left\{{ }^{1} \mathrm{H}\right\}$ NMR $\delta$ 32.7, 33.3, 33.5; MS (MALDI-TOF) $m / z$ z $618\left(\mathrm{M}^{+}\right)$.

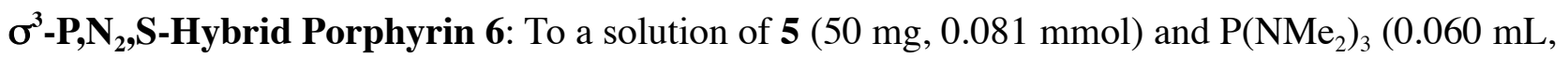
$0.32 \mathrm{mmol})$ in degassed toluene $(3 \mathrm{~mL})$, was added a toluene $(2 \mathrm{~mL})$ solution of DDQ (130 mg, 0.57 $\mathrm{mmol}$ ) over $40 \mathrm{~min}$ at $-78{ }^{\circ} \mathrm{C}$, and the resulting mixture was directly subjected to a short alumina column $\left(\mathrm{CH}_{2} \mathrm{Cl}_{2}\right)$. Further purification by alumina column chromatography, followed by recrystallization from $\mathrm{CH}_{2} \mathrm{Cl}_{2} / \mathrm{MeOH}$, afforded 6 as a reddish purple solid (7 mg, 15\%): $\mathrm{Mp} \mathrm{ca} .250{ }^{\circ} \mathrm{C}$ (decomp); ${ }^{1} \mathrm{H}$ NMR $\delta 2.29$ (dd, $\left.J=7.6 \mathrm{~Hz},{ }^{3} J_{\mathrm{P}-\mathrm{H}}=4.5 \mathrm{~Hz}, 2 \mathrm{H}\right), 2.90-3.00(\mathrm{~m}, 1 \mathrm{H}), 3.20-3.31(\mathrm{~m}, 1 \mathrm{H})$, 3.53-3.59 (m, 2H), 4.40-4.51 (m, 2H), 5.27 (dd, J=7.6 Hz, 7.2 Hz, 2H), $5.66(\mathrm{t}, J=7.2 \mathrm{~Hz}, 1 \mathrm{H}), 7.75$ (br, 6H), $7.91(\mathrm{br}, 2 \mathrm{H}), 8.33(\mathrm{br}, 2 \mathrm{H}), 8,67(\mathrm{~d}, J=4.4 \mathrm{~Hz}, 2 \mathrm{H}), 8.93(\mathrm{~d}, J=4.4 \mathrm{~Hz}, 2 \mathrm{H}), 9.22(\mathrm{~s}, 2 \mathrm{H})$, $10.44\left(\mathrm{~d},{ }^{3} J_{\mathrm{P}-\mathrm{H}}=18.8 \mathrm{~Hz}, 2 \mathrm{H}\right) ;{ }^{31} \mathrm{P}\left\{{ }^{1} \mathrm{H}\right\} \mathrm{NMR} \delta 18.6 ; \mathrm{UV} / \mathrm{Vis}\left(\mathrm{CH}_{2} \mathrm{Cl}_{2}\right) \lambda_{\max }(\varepsilon): 440(141000), 492$ (11600), 518 (7400), 547 (4900), 647 (1300), 718 (3100); MS (FAB) m/z $613\left([\mathrm{M}+\mathrm{H}]^{+}\right)$. HR-FAB-MS: Calcd for $\mathrm{C}_{41} \mathrm{H}_{29} \mathrm{~N}_{2} \mathrm{PS}\left(\mathrm{M}^{+}\right), 612.1789$; Found, 612.1788. 


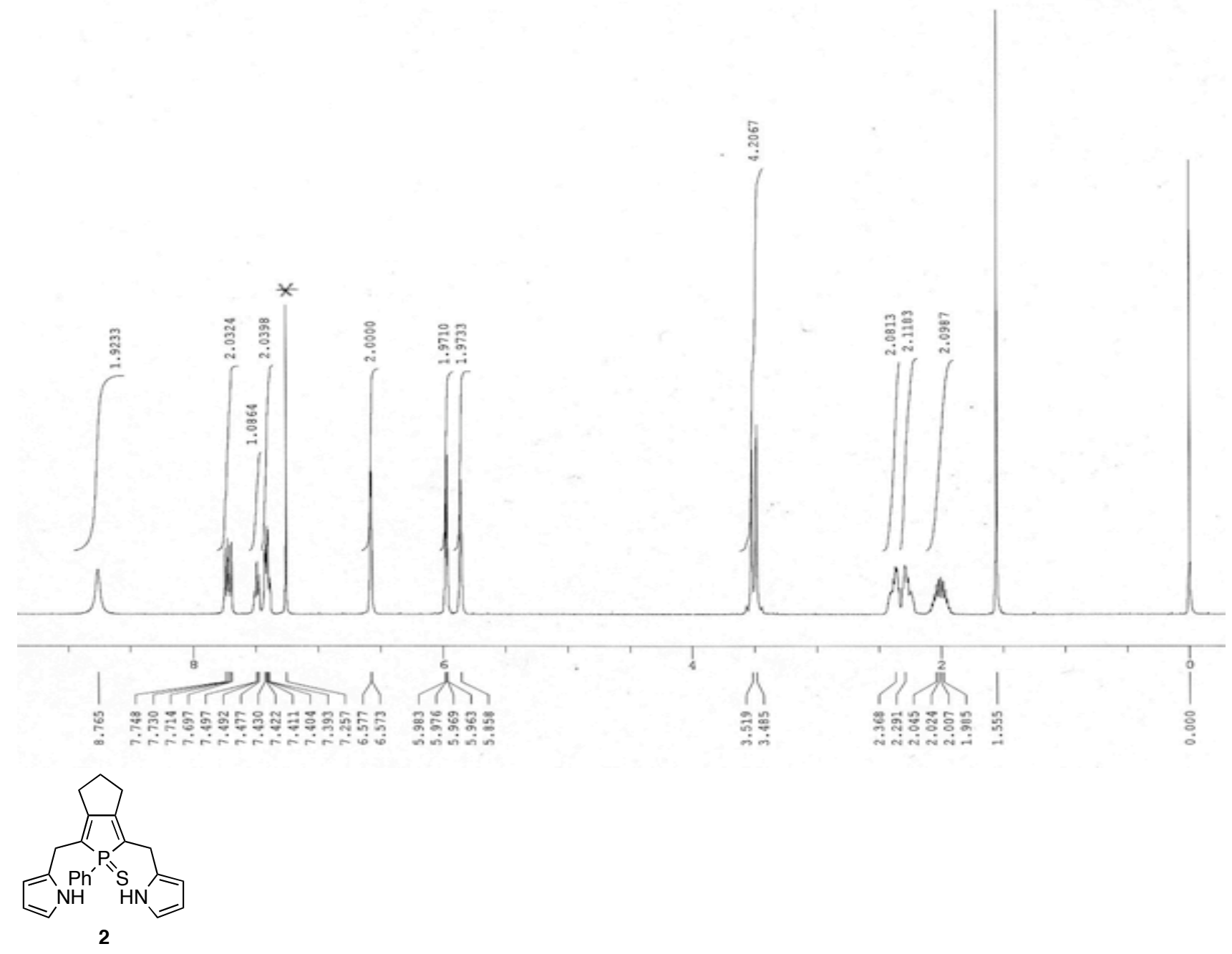

Figure S1. ${ }^{1} \mathrm{H}$ NMR spectrum of 2 in $\mathrm{CDCl}_{3}$. 


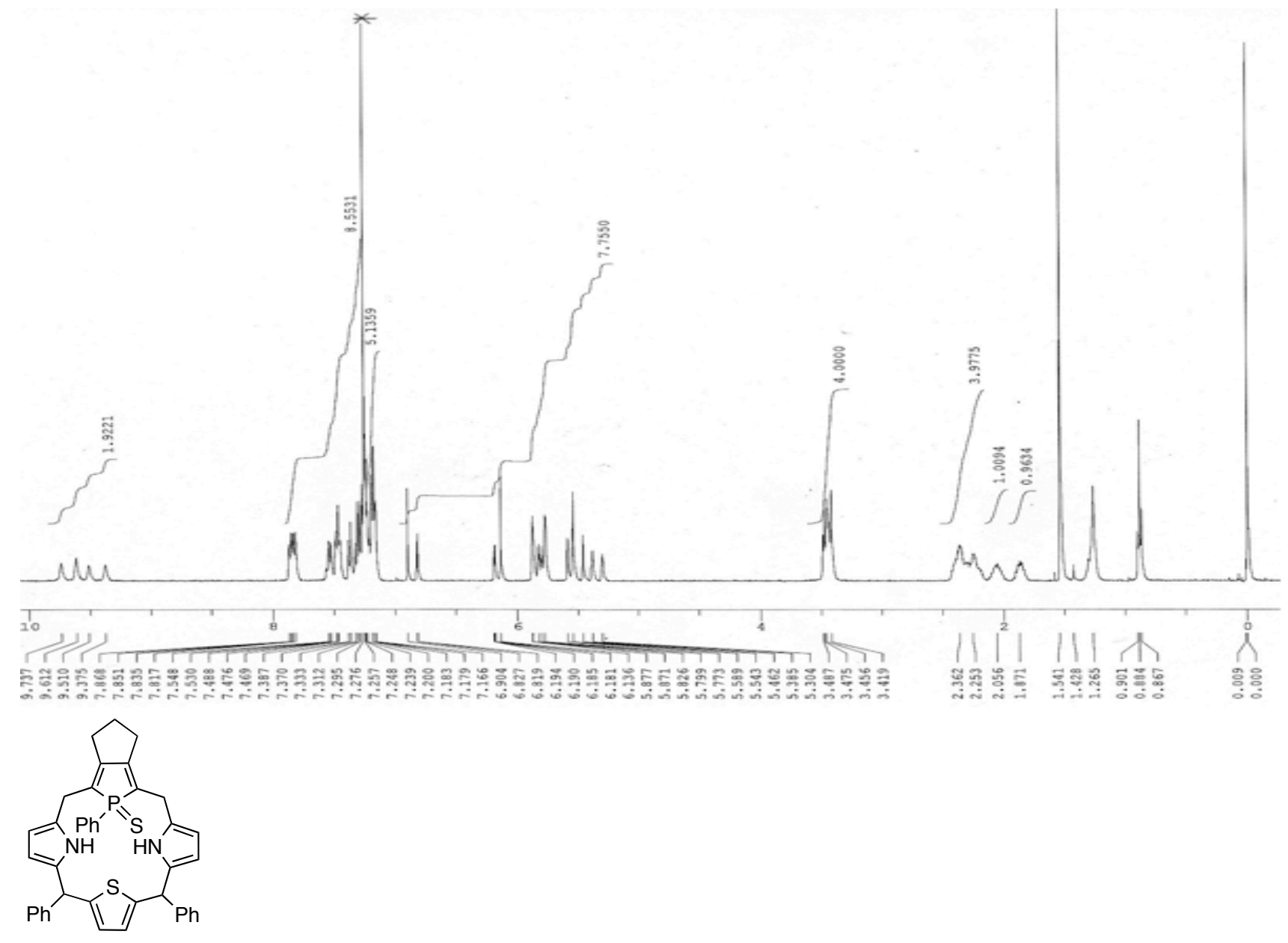

Figure S2. ${ }^{1} \mathrm{H}$ NMR spectrum of 4 in $\mathrm{CDCl}_{3}$. 


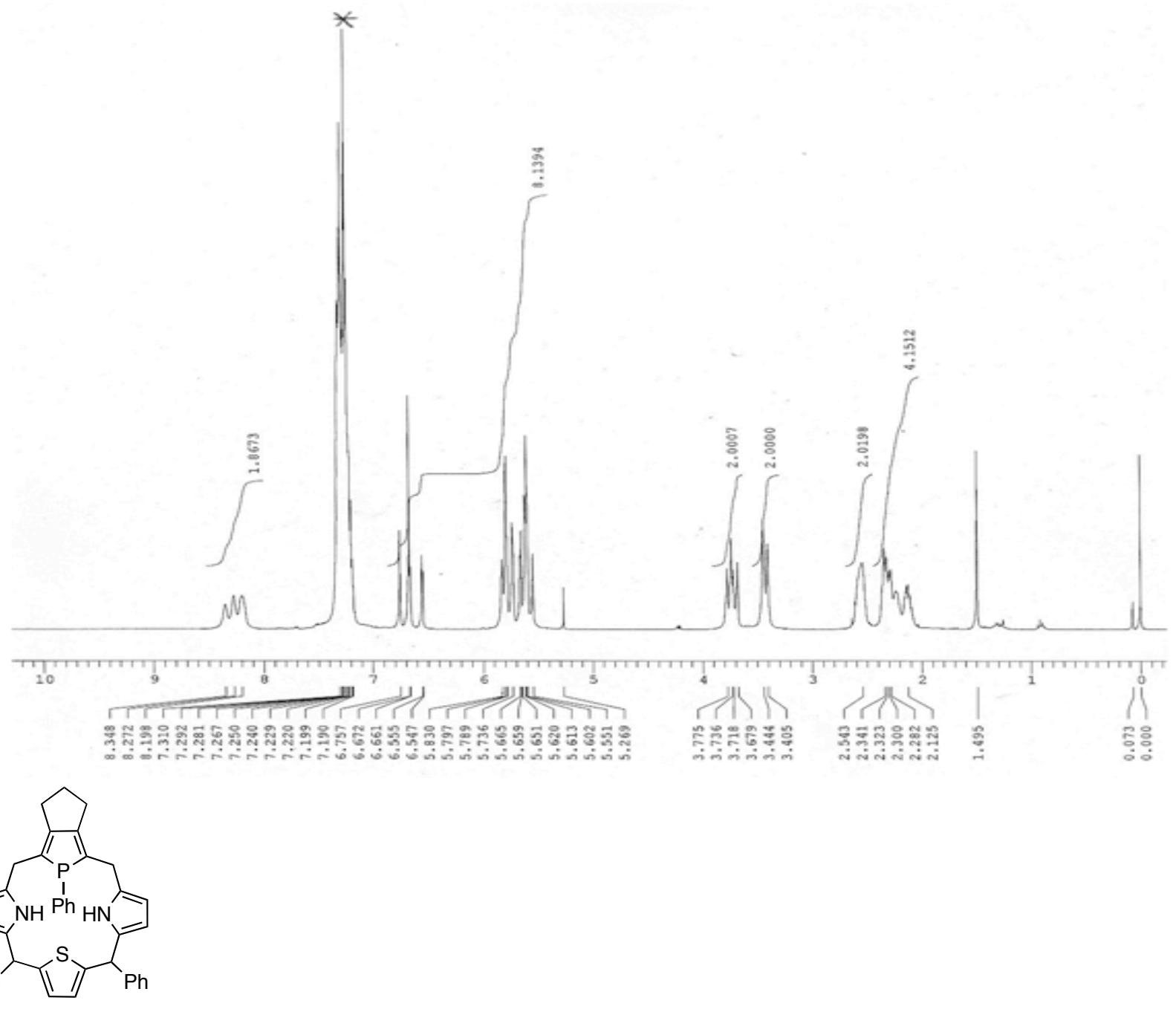

Figure S3. ${ }^{1} \mathrm{H}$ NMR spectrum of 5 in $\mathrm{CDCl}_{3}$. 


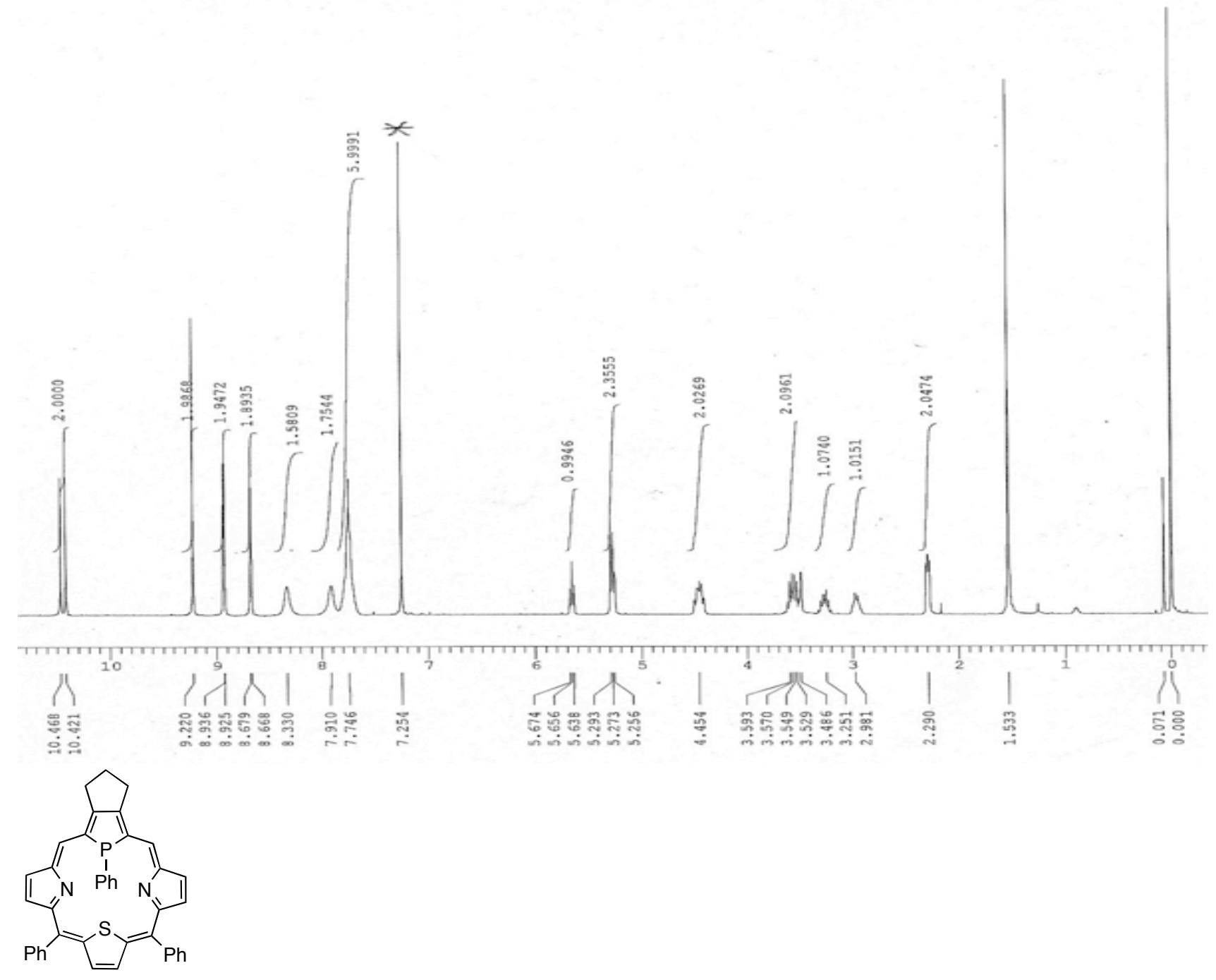

Figure S4. ${ }^{1} \mathrm{H}$ NMR spectrum of 6 in $\mathrm{CDCl}_{3}$. 


\section{Density Functional Theory (DFT) Calculations on Model Compounds}

The structures of $\sigma^{3}-\mathrm{P}, \mathrm{N}_{2}, \mathrm{~S}$-hybrid 10, parent porphyrin $\mathbf{8 b}$, and 21-thiaporphyrin $\mathbf{9 b}$, as models for $\mathbf{6 , 8 a}$, and 9a, respectively, were optimized using density functional theory (DFT). The basis set used was 6-311G(d,p). ${ }^{3}$ The functionals of DFT was the Becke 1988 exchange and Lee-Yang-Parr correlation functionals (B3LYP). ${ }^{4}$ The nucleus independent chemical shift (NICS) values ${ }^{5}[$ NICS(0) values] were calculated at the Hartree-Fock level with gauge-including atomic orbitals (GIAOs) at the DFT optimized structures. The basis set used in the NICS value computations was $6-31+\mathrm{G}(\mathrm{d}){ }^{6}{ }^{6}$ The optimized structures are depicted in Figure S5, and the bond lengths of the optimized structures are shown in Figure S6. All the calculations were carried out using the Gaussian 03 suite of programs. ${ }^{7}$

3 (a) Krishnan, R.; Binkley, J. S.; Seeger, R.; Pople, J. A. J. Chem. Phys. 1980, 72, 650. (b) McLean, A. D.; Chandler, G. S. J. Chem. Phys. 1980, 72, 5639.

${ }^{4}$ (a) Becke, A. D. J. Chem. Phys. 1988, 98, 5648. (b) Lee, C.; Yang, W.; Parr, R. G. Phys. Rev. B 1988, 37, 785 .

${ }^{5}$ Schleyer, P. v. R.; Maerker, C.; Dransfeld, A.; Jiao, H.; van Eikema Hommes, N. J. R. J. Am. Chem. Soc. 1996, 118, 6317.

${ }^{6}$ (a) Hehre, W. J.; Ditchfield, R.; Pople, J. A. J. Chem. Phys. 1972, 56, 2257. (b) Francl, M. M.; Pietro, W. J.; Hehre, W. J.; Binkley, J. S.; DeFrees, D. J.; Pople, J. A.; Gordon, M. S. J. Chem. Phys. 1982, 77, 3654. (c) Clark, T.; Chandrasekhar, J.; Spitznagel, G. W.; Schleyer, P. von R. J. Comp. Chem. 1983, 4, 294.

${ }^{7}$ Frisch, M. J.; Trucks, G. W.; Schlegel, H. B.; Scuseria, G. E.; Robb, M. A.; Cheeseman, J. R.; Montgomery, J. A. Jr.; Vreven, T.; Kudin, K. N.; Burant, J. C.; Millam, J. M.; Iyengar, S. S.; Tomasi, J.; Barone, V.; Mennucci, B.; Cossi, M.; Scalmani, G.; Rega, N.; Petersson, G. A.; Nakatsuji, H.; Hada, M.; Ehara, M.; Toyota, K.; Fukuda, R.; Hasegawa, J.; Ishida, M.; Nakajima, T.; Honda, Y.; Kitao, O.; Nakai, H.; Klene, M.; Li, X.; Knox, J. E.; Hratchian, H. P.; Cross, J. B.; Adamo, C.; Jaramillo, J.; Gomperts, R.; Stratmann, R. E.; Yazyev, O.; Austin, A. J.; Cammi, R.; Pomelli, C.; Ochterski, J. W.; Ayala, P. Y.; Morokuma, K.; Voth, G. A.; Salvador, P.; Dannenberg, J. J.; Zakrzewski, V. G.; Dapprich, S.; Daniels, A. D.; Strain, M. C.; Farkas, O.; Malick, D. K.; Rabuck, A. D.; Raghavachari, K.; Foresman, J. B.; Ortiz, J. V.; Cui, Q.; Baboul, A. G.; Clifford, S.; Cioslowski, J.; Stefanov, B. B.; Liu, G.; Liashenko, A.; Piskorz, P.; Komaromi, I.; Martin, R.L.; Fox, D. J.; Keith, T.; Al-Laham, M. A.; Peng, C.Y.; Nanayakkara, A.; Challacombe, M.; Gill, P. M. W.; Johnson, B.; Chen, W.; Wong, M. W.; Gonzalez, C.; Pople, J. A. Gaussian 03; Gaussian, Inc.: Pittsburgh PA, 2003. 
(a)

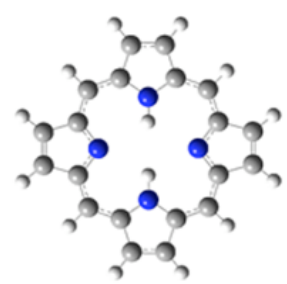



(b)

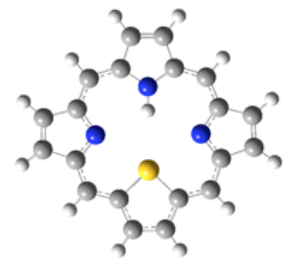

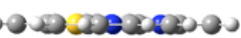

(c)
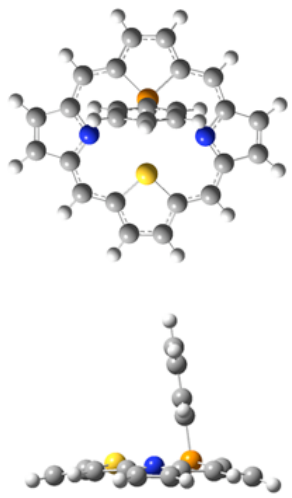

Figure S5. Top (upper) and side (lower) views of the optimized structures of (a) $\mathbf{8 b}$, (b) $\mathbf{9 b}$, and (c) 10. The $\mathrm{C}, \mathrm{N}, \mathrm{P}$, and $\mathrm{S}$ atoms are indicated as grey, blue, orange, and yellow balls, respectively.

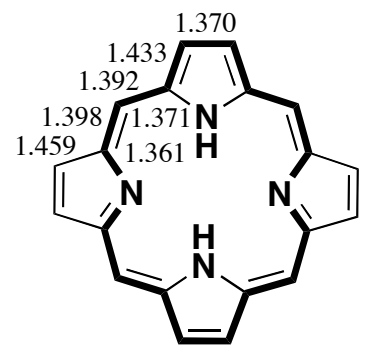

8b

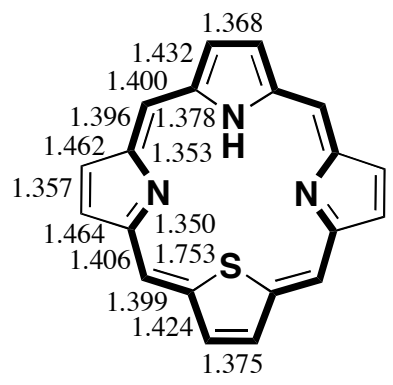

9b

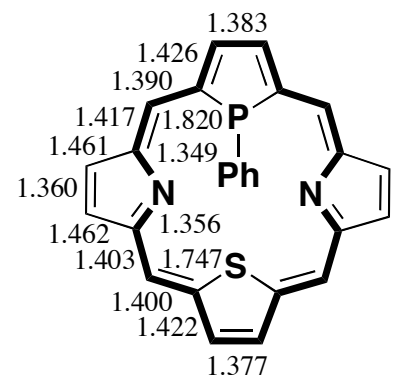

10

Figure S6. Bond lengths $(\AA)$ of the optimized structures of $\mathbf{8 b}, \mathbf{9 b}$, and $\mathbf{1 0}$. 